\title{
The efficacy of dydrogesterone use to suppress premature luteinizing hormone surge on cycle outcomes in controlled ovarian stimulation
}

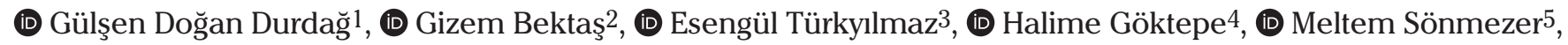 \\ (D) Yavuz Emre Şükür6, (D) Batuhan Özmen 6 , (D) Cem Atabekoğlu6, (D) Bülent Berker6, (D) Ruşen Aytaç6, \\ (D) Murat Sönmezer6 \\ 1Department of Obstetrics and Gynecology, Başkent University, Adana Dr. Turgut Noyan Application and Research Center, \\ Adana, Turkey \\ 2Gürgan Clinic, Ankara, Turkey \\ ${ }^{3}$ Clinic of Obstetrics and Gynecology, Ankara City Hospital, Ankara, Turkey \\ ${ }^{4}$ Clinic of Obstetrics and Gynecology, Konya Training and Research Hospital, Konya, Turkey \\ 5Private Clinic, Ankara, Turkey \\ 6Department of Obstetrics and Gynecology, Ankara University Faculty of Medicine, Ankara, Turkey
}

\section{Abstract}

Objective: Progestins are used as an alternative to gonadotropin releasing hormone (GnRH) antagonists to suppress premature luteinizing hormone (LH) surge and a flexible protocol has been defined recently. The aim of this study was to compare the efficacy of flexible protocols with dydrogesterone and GnRH antagonist in suppressing LH surge.

Material and Methods: This retrospective, case-control study, was conducted in an infertility unit of a tertiary university hospital. A daily dose of $40 \mathrm{mg}$ dydrogesterone was compared with GnRH antagonist (GnRHant) in controlled ovarian hyperstimulation cycles between July 2018 and July 2019. Dydrogesterone was started when the leading follicle was $12 \mathrm{~mm}$ or serum estradiol was over $300 \mathrm{pg} / \mathrm{mL}$. A subgroup analysis of poor responder patients was also performed.

Results: In total there were 105 subjects aged between 23 and 41 years, 52 in the dydrogesterone group and 53 in the GnRHant group. Duration of pituitary suppression was longer in dydrogesterone group. Premature ovulation was observed in $11.5 \%$ (6/52) and $0 \%$ in the dydrogesterone and GnRHant groups, respectively. However, collected oocyte counts and metaphase II oocyte counts were found to be similar between the groups. The six patients with premature ovulation were in poor responder subgroup.

Conclusion: Dydrogesterone can be used as an alternative to antagonist regimen in patients where embryo transfer is not planned in the same cycle. However, flexible regimen may not be appropriate in patients with diminished ovarian reserve, as advanced follicular maturation and delayed suppressive effect of oral progesterone may cause premature ovulation. Randomized controlled trials in particular patient groups are required to determine the most effective minimum dose and time of application to ensure treatment success. (J Turk Ger Gynecol Assoc 2021; 22: 293-9)

Keywords: Controlled ovarian hyperstimulation, dydrogesterone, luteinizing hormone surge, progestin-primed ovarian stimulation

Received: 1 July, 2020 Accepted: 09 December, 2020 


\section{Introduction}

In controlled ovarian hyperstimulation $(\mathrm{COH})$ cycles, the aim is to develop numerous follicles to obtain an optimum number and quality of oocytes. Premature luteinizing hormone (LH) surge and premature ovulation during the treatment is one of the major causes of cycle cancellation (1). In gonadotropin releasing hormone $(\mathrm{GnRH})$ antagonist protocol, $\mathrm{GnRH}$ antagonists (GnRHant) suppress endogenous gonadotropins in minutes. However, new cheaper stimulation regimens that do not compromise success rates are under investigation $(2,3)$.

Studies have demonstrated that progesterone is an important regulator on timing of ovulation, and it can be used instead of GnRHant to prevent early luteinization. In the protocol, which is defined as progestin-primed ovarian stimulation (PPOS), progestins are used as an alternative to GnRHant to suppress LH surge. For this purpose, various progesterone forms and doses have been reported (4). Progesterone treatment controls oocyte development and timing of ovulation but, owing to disruption of synchronization between embryo development and endometrial receptivity due to untimely progesterone exposure, embryos obtained from these oocytes cannot be transferred in the same cycle (5). Nevertheless, improvements in cryopreservation and vitrification techniques enable the freezing of all oocytes or embryos to be transferred in subsequent cycles $(2,6)$.

A few studies have shown that the application of this protocol does not adversely affect oocyte development and the number of oocytes obtained, compared to antagonist protocol $(1,7,8)$. Besides, a flexible PPOS protocol has been defined recently (9).

The aim of this study was to investigate whether a synthetic progesterone, dydrogesterone, when used in a flexible protocol, is as effective as GnRHant in suppressing the LH surge. The effect of dydrogesterone on other cycle parameters was also investigated.

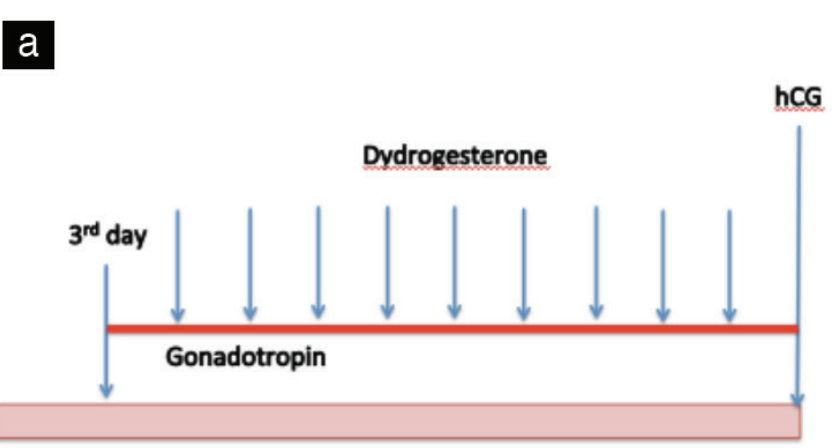

\section{Material and Methods}

This retrospective, case-control study was conducted in an infertility unit of a tertiary referral university hospital, involving patients aged between 23 and 41 years, for whom GnRHant or oral dydrogesterone was used to block premature LH surge in COH cycles between July 2018 and July 2019. Patients who applied for fertility preservation due to advanced age or malignancy, or who underwent controlled ovarian stimulation due to diminished ovarian reserve, unexplained infertility, endometriosis or male factor were included in the study.

In dydrogesterone group, starting from the third day of the cycle, 150-225 IU gonadotropin, either human menopausal gonadotropin (hMG) (Merional ${ }^{\circledR}$ 75IU, IBSA Institut) or recombinant follicle stimulating hormone $(\mathrm{rFSH})$ (Gonal- $\mathrm{f}^{\circledR}$ 75IU, Merck-Serono) was administered. Dydrogesterone (Duphaston ${ }^{\circledR} 10 \mathrm{mg}$, Abbott Farma, Netherlands) 2x20 mg/ day was started when the dominant follicle reached 12 $\mathrm{mm}$ in diameter or serum estradiol was over $300 \mathrm{pg} / \mathrm{mL}$. Gonadotropin dose was adjusted according to the response of the ovary five days later and it was used until trigger day, while dydrogesterone was continued at a dose of 2x20 mg until trigger day. When two or more follicles reached $18 \mathrm{~mm}$ diameter, final oocyte maturation was triggered with $250 \mu \mathrm{g}$ choriogonadotropin alpha (Ovitrelle ${ }^{\circledR} 250$ mcg, Merck-Serono) and GnRHant (Decapeptyl ${ }^{\circledR} 0.1 \mathrm{mg}$, Ipsen Pharma or Lucrin ${ }^{\circledR}$ $5 \mathrm{mg} / \mathrm{mL}$, Abbott). Regular and flexible PPOS protocols are shown in Figure 1a, b.

The control group consisted of age-matched patients, who received the GnRHant, cetrorelix (Cetrotide ${ }^{\circledR} 0.25$ mg, MerckSerono) by antagonist protocol. This group was given 150-225 IU gonadotropin, either hMG or $\mathrm{rFSH}$, as detailed above. When the dominant follicle reached $13 \mathrm{~mm}$ in diameter, $0.25 \mathrm{mg}$ cetrorelix was started and continued until trigger day.

Gonadotropin dose was also adjusted according to ovarian response and it was continued until trigger day. When two or more follicles reached $18 \mathrm{~mm}$ in diameter, final oocyte b

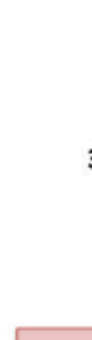

$12 \mathrm{~mm}$ follicle

or
hCG

Figure 1. (a) Regular PPOS protocol, (b) Flexible PPOS protocol

PPOs: Progestin-primed ovarian stimulation 
maturation was triggered with $250 \mu$ g choriogonadotropin alpha (Ovitrelle ${ }^{\circledR} 250$ mcg, Merck-Serono) and GnRHant (Decapeptyl ${ }^{\circledR}$ $0.1 \mathrm{mg}$, Ipsen Pharma or Lucrin ${ }^{\circledast} 5 \mathrm{mg} / \mathrm{mL}$, Abbott). In both groups oocyte retrieval was performed 36 hours after trigger application. Cryopreservation of the oocytes was performed using a vitrification technique.

Patients' records were examined and data items including age, body mass index, cause of infertility, type of $\mathrm{COH}$ protocol, duration and total dose of gonadotropins, duration of antagonist (cetrorelix)/dydrogesterone use, basal hormone levels, the suppression of premature LH surge, premature ovulation, and total and mature oocyte counts were collected and evaluated. Premature LH surge was defined as serum $\mathrm{LH}$ level $>15 \mathrm{mIU} / \mathrm{mL}$ on trigger day (8). Premature ovulation was defined as rupture of the dominant follicle before oocyte retrieval and elevation of serum progesterone $>3 \mathrm{ng} / \mathrm{mL}$ (8). Efficacy in suppressing premature LH surge was compared between oral dydrogesterone and GnRH antagonist.

A subgroup analysis of poor responder patients in both the study and control groups was further performed. This subgroup included patients belonging to group 3 and 4 according to Poseidon classification, that is patients with anti-mullerian hormone (AMH) $<1.2 \mathrm{ng} / \mathrm{mL}$ or antral follicle count $<5$ (10).

This study was approved by Institutional Review Board and Ethics Committee of Ankara University Faculty of Medicine (approval number: 20-1364-18). This study was performed in accordance with Declaration of Helsinki. All patients gave informed consent prior to their treatment.

\section{Statistical analyses}

Data analyses were performed using SPSS, version 21.0 (IBM Corporation, Armonk, NY, USA). The variables were investigated using visual (histograms and probability plots) and analytical methods (Shapiro-Wilk test) to determine whether or not they were normally distributed. As data was non-normally distributed, non-parametric tests were used. Descriptive statistics of continuous variables were compared between groups using Mann-Whitney $U$ test. The chi-square test or Fisher's exact test (when chi-square test assumptions do not hold due to low expected cell counts) were used to compare categorical variables between groups. Continuous variables are presented as median and interquartile range limiting the reported range to values between the $25-75^{\text {th }}$ percentiles, whereas categorical variables are presented as number and percentage. A p $<0.05$ was considered statistically significant.

\section{Results}

In total 105 women participated in the study, of whom 52 were included in the dydrogesterone group and 53 in the GnRHant group. Demographic features, total duration and dose of gonadotropins, number of total oocytes and metaphase II oocytes collected were similar between the two groups (Table 1). However, total duration of dydrogesterone/cetrorelix administration was found to be significantly different $(\mathrm{p}<0.001)$. Trigger day estradiol was lower, while trigger day progesterone and maximum LH levels were higher in the dydrogesterone group. It was also remarkable that AMH levels were similarly low in both groups [0.80 (0.38-2.31) and $0.42(0.30-4.0) \mathrm{ng} /$ $\mathrm{mL}$ in the dydrogesterone and antagonist groups, respectively $(\mathrm{p}=0.188)]$.

Indications for $\mathrm{COH}$ were fertility preservation due to advanced age in 28 (26.7\%) patients, fertility preservation due to malignancy in $12(11.4 \%)$ patients, diminished ovarian reserve in $29(27.6 \%)$ patients, unexplained infertility in

Table 1. Demographic and clinical features of the groups

\begin{tabular}{|l|l|l|l|}
\hline & Dydrogesterone (n=52) & Cetrorelix (n=53) & p \\
\hline Median (IQR) age (years) & $33(25-38)$ & $32(30-36)$ & $25.8(23.3-28.6)$ \\
\hline Median (IQR) BMI & $24.1(19.7-25.4)$ & $0(0-0)$ & 0.103 \\
\hline Median (IQR) parity & $0(0-0)$ & $0.42(0.30-4.0)$ & 0.476 \\
\hline Median (IQR) AMH (ng/mL) & $0.80(0.38-2.31)$ & $4(3-6)$ & 0.188 \\
\hline Median (IQR) duration of pituitary suppression (days) & $6(4-7)$ & $9(8-11)$ & $1950(1560-2712)$ \\
\hline Median (IQR) duration of gonadotropins (days) & $10(8-11)$ & $1395(550-2382)$ & 0.110 \\
\hline Median (IQR) total dose of gonadotropins (IU) & $2025(1800-2475)$ & $1(0.80-7.75)$ \\
\hline Median (IQR) trigger day E (pg/mL) & $748(150-1060)$ & $4(2.5-16)$ \\
\hline Median (IQR) trigger day P (ng/mL) & $1.27(1.0-2.29)$ & $8(2-13)$ & $\mathbf{0 . 0 0 7}$ \\
\hline Median (IQR) maximum LH (mIU/mL) & $6(5.0-10.47)$ & $7(1-11)$ & $\mathbf{0 . 0 0 4}$ \\
\hline Median (IQR) number of oocytes & $8(2-12)$ & $0(0)$ & 0.005 \\
\hline Median (IQR) number of MII oocytes & $6(1-10)$ & $6(11.5)$ & 0.399 \\
\hline Premature ovulation, $n$ (\%) & 0.013 \\
\hline BMI: Body mass index, AMH: Anti-mullerian hormone, $\mathrm{E}_{2}$ : Estradiol, P: Progesterone, LH: Luteinizing hormone, IQR: Interquartile range, MII: Metaphase II \\
\hline
\end{tabular}


$3(2.9 \%)$ patients, endometriosis in $14(13.3 \%)$ patients, and male factor in 19 (18.1\%) patients (Table 2). There was no difference between the dydrogesterone and GnRHant groups in terms of indications $(p=0.215)$.

Dual trigger was used in all patients, with the exception of five patients in whom estradiol was $>4000 \mathrm{pg} / \mathrm{mL}$ on trigger day, when analog trigger was applied. The two groups were also similar with respect to application of trigger agents.

Premature LH surge was present in 13.5\% in dydrogesterone group and in $9.4 \%$ of the antagonist group, which was not significant $(p=0.517)$. However, premature ovulation before the scheduled oocyte pick up day occurred in $6(11.5 \%)$ patients in dydrogesterone group, whereas it did not occur in antagonist group ( $p=0.013)$. In four of these six patients, oocytes could be collected from the other follicles, while oocyte retrieval failed in the remaining two patients due to diminished ovarian reserve. The subgroup of poor responder patients included 40 patients, of whom $25(62.5 \%)$ were in the dydrogesterone group and 15 (37.5\%) were in the antagonist group. Clinical outcomes were similar poor responders in both groups but all six patients who had premature ovulation were in the dydrogesterone group (Table 3).

\section{Discussion}

This study demonstrated that dydrogesterone, used in a flexible PPOS protocol, provided similar results in some respects to antagonist protocol in preventing premature LH surge. Favorable results included total number and quality of oocytes collected. However, a high incidence of premature ovulation in patients receiving dydrogesterone suggested that flexible regimen might not be suitable in all patients.

Transient, but quick, LH suppression provided by GnRHant is associated with competitive blockade of $\mathrm{GnRH}$ receptors $(8,11)$, while progestins suppress $\mathrm{GnRH}$ secretion at the hypothalamus when they are administered in the early phase of the cycle and prior to estrogen elevation $(8,12)$. It has been stated that serum LH levels are more stable with PPOS and oocyte retrieval can be planned more precisely (8). Furthermore, ease of oral use instead of daily injections and lower cost of treatment are advantageous $(7,9,13)$. However, as the main limitation of these protocols is the inability to perform transfer in the same cycle, progestins may be more suitable for planned freeze-all cycles, pre-implantation genetic testing cycles, elective oocyte cryopreservation and oocyte donor stimulation (14).

Table 2. Indications of controlled ovarian hyperstimulation

\begin{tabular}{|l|l|l|l|}
\hline & Dydrogesterone (n, \%) & Cetrorelix (n, \%) & Total number of patients \\
\hline Fertility preservation due to advanced age & $12(42.9 \%)$ & $16(57.1 \%)$ & 28 \\
\hline Fertility preservation due to malignancy & $9(75.0 \%)$ & $3(25.0 \%)$ & 12 \\
\hline Diminished ovarian reserve & $16(55.2 \%)$ & $13(44.8 \%)$ & 29 \\
\hline Unexplained infertility & $1(33.3 \%)$ & $2(66.7 \%)$ & 3 \\
\hline Endometriosis & $8(57.1 \%)$ & $6(42.9 \%)$ & 14 \\
\hline Male factor & $6(31.6 \%)$ & $13(68.4 \%)$ & 19 \\
\hline
\end{tabular}

Table 3. Demographic and clinical features of the patients in the poor responder subgroup

\begin{tabular}{|c|c|c|c|}
\hline & $\begin{array}{l}\text { Dydrogesterone } \\
(n=25)(48.1 \%)\end{array}$ & $\begin{array}{l}\text { Cetrorelix }(n=15) \\
(28.3 \%)\end{array}$ & $\mathbf{p}$ \\
\hline Median (IQR) age (years) & $32(25-38)$ & $35(32-37)$ & 0.595 \\
\hline Median (IQR) parity & $0(0-0)$ & $0(0-0)$ & 0.289 \\
\hline Median (IQR) AMH (ng/mL) & $0.34(0.09-0.74)$ & $0.42(0.22-0.58)$ & 0.903 \\
\hline Median (IQR) total dose of gonadotropins (IU) & $1800(1425-2025)$ & $2213(1631-3600)$ & 0.345 \\
\hline Median (IQR) trigger day $\mathrm{E}_{2}(\mathrm{pg} / \mathrm{mL})$ & $157(78-724)$ & $408(335-1132)$ & 0.108 \\
\hline Median (IQR) trigger day $\mathrm{P}(\mathrm{ng} / \mathrm{mL})$ & $1.0(0.6-1.5)$ & $5.0(1.0-9.5)$ & 0.054 \\
\hline Median (IQR) maximum LH (mIU/mL) & $9.0(6.3-14.3)$ & $11.5(7.5-17.0)$ & 0.897 \\
\hline
\end{tabular}


Progesterone is known to inhibit estradiol-induced LH surge, both in early follicular phase and early luteal phase (1). Although there is much concerning endogenous LH surge and the role of progesterone to be elucidated (15), it has been reported that progesterone should be administered at the right time to be effective (1). Recently, multiple follicle selection waves and random start protocols have brought attention to flexible PPOS programs, and in a study, including donor cycles, it was shown that a flexible PPOS protocol can effectively suppress premature ovulation as well (9).

In our study, cycle parameters other than the incidence of early ovulation were mostly similar between the groups. Notably, total duration of pituitary suppression was longer in dydrogesterone group, since we started to use dydrogesterone one day earlier than is normal with GnRHant administration protocols and this result is similar to some previous studies. In the report of Kuang et al. (1), while hMG dose and duration were higher in the study group, collected oocyte counts and other cycle parameters were similar. In the study of Xiao et al. (7), while dose and duration of gonadotropins were higher in the PPOS group, other characteristics were similar. Cycle parameters were similar in the study of Chen et al. (8), and were also similar in the study of Wang et al. (16), with the exception of hMG dose being higher in the PPOS group. Lower trigger day estradiol levels in the dydrogesterone group in our study were probably caused by a higher number of poor responder patients $(25 / 52)$ in this group. Also, the difference between LH levels, which were $<10 \mathrm{mIU} /$ $\mathrm{mL}$ in both groups, was not considered clinically significant, the higher trigger day progesterone levels in our dydrogesterone group were consistent with the finding of premature ovulation. Patients who underwent $\mathrm{COH}$ for a range of indications were included in our study. Data regarding different patient groups using PPOS protocols are available in the literature. PPOS is reported to be successful in polycystic ovary syndrome (PCOS) patients as an alternative to the antagonist protocol, as it reduces the risk of ovarian hyperstimulation syndrome, suppresses premature LH surge, and uses a freeze-all strategy $(7,16)$. It has been suggested that higher total gonadotropin dose and duration with PPOS are caused by decreased follicle sensitivity due to high progesterone and pituitary suppression (7). On the other hand, there are also studies which report that PPOS suppresses LH surge better than GnRHant, with similar oocyte counts, in poor responder patients $(8,17,18)$. Among our patients who received treatment for fertility preservation due to malignancy, PPOS protocol was not administered to patients with breast cancer, while two of the patients in the cetrorelix group had breast cancer. These two patients received letrozole $5 \mathrm{mg} /$ day (Femara ${ }^{\circledR}$, Novartis, Switzerland), starting from the third day of the menstrual cycle along with gonadotropin, until trigger day.
Synthetic progesterones are preferred in studies as natural micronized progesterones can affect serum values, and medroxyprogesterone acetate (MPA) is the most commonly used agent for this purpose. Kuang et al. (1) compared $10 \mathrm{mg} /$ day MPA with standard antagonist protocol for the first time, and found similar results in terms of hMG dose and duration, as well as oocyte and embryo counts. It was previously reported that MPA could not inhibit ovulation at a dose of $5 \mathrm{mg} /$ day (19). However, Dong et al. (20), who investigated the minimum dose to suppress LH surge, concluded that $4 \mathrm{mg}$ MPA was similar to $10 \mathrm{mg}$ in terms of the number of oocytes collected and was sufficient to prevent premature LH surge. Yu et al. (4) compared MPA and dydrogesterone to suppress premature LH surge, and reported that premature LH surge was not seen in either group, and similar oocyte counts and cycle characteristics were observed between the groups. Nevertheless, there are few studies using dydrogesterone, and no exact protocol in terms of dose and duration has been specified. There are reports that dydrogesterone does not prevent ovulation at recommended doses (10-20 mg/day) for MPA, and a minimum dose of $30 \mathrm{mg}$ dydrogesterone is required for this purpose $(21,22)$. Yu et al. (4) also concluded that dydrogesterone is less proficient than MPA in suppressing GnRH, and a minimum $20 \mathrm{mg}$ dose is needed to be effective. Based on these reports, while $30 \mathrm{mg} /$ day is considered to be a suitable dose for dydrogesterone, in order to provide patient compliance, $40 \mathrm{mg} /$ day (2x2 tablets) dydrogesterone was preferred in our study.

Rates of premature LH surge were $13.5 \%$ and $9.4 \%$ in dydrogesterone and cetrorelix groups respectively, which was not significantly different. In the study of Kuang et al. (1), premature LH surge was not observed in either group, and only occurred in 1 in 150 of the whole cohort. In the study of Chen et al. (8) in the poor responder group, the rate of premature $\mathrm{LH}$ surge was significantly lower in the PPOS group compared to the antagonist group. However, the number of obtained oocytes and embryos were similar. In the study of Wang et al. (16) in the PCOS group, premature LH surge and premature ovulation were not reported, and the cycle parameters were similar, with the exception of higher hMG doses in the MPA group. However, there are also studies reporting higher rates of premature $\mathrm{LH}$ surge. Although the suppressive effect of GnRHant on LH is rapid and reversible, premature LH surge is reported but is extremely variable at $0.34-38 \%$ of the patients $(23,24)$. When compared to the prompt effect of GnRHant, dydrogesterone acts more slowly as its peak plasma level is achieved after one hour (21).

Collection of oocytes before ovulation in some of our patients with premature LH surge was accomplished by changing the oocyte aspiration time, based on LH monitoring. This positive effect of LH monitoring has also been reported in previous 
studies. Chen et al. (8), in their study with poor responders, observed that at least one mature oocyte could be collected from 9 of 10 patients who had premature LH surge in the antagonist group by changing the oocyte retrieval time. $\mathrm{LH}$ monitoring was not performed in the flexible PPOS protocol study of Yildiz et al. (9) but premature ovulation was not reported for either group, though this study included donor cycles and not poor responders.

In our study, dual trigger was used for completion of final maturation, in line with the results of previous studies $(1,16,25)$. While premature ovulation was not observed in our cetrorelix group, this was observed in the dydrogesterone group, which was a significant difference. It was formerly reported that, rise in serum progesterone level after the increase in serum estradiol concentrations results in earlier LH surge $(26,27)$. Therefore, higher premature LH surge and premature ovulation rates in our dydrogesterone group may be related to the late administration of dydrogesterone in our study, when compared to these earlier studies, in which progesterone administration was started at the third day of the cycle $(1,4,8)$. It is also reported that diminished ovarian reserve increases the risk of premature LH surge in antagonist cycles (24). Although we classified our cohort due to $\mathrm{COH}$ indications, $48.1 \%$ and $28.3 \%$ of the patients were poor responders in dydrogesterone and $\mathrm{GnRH}$ antagonist groups respectively. It has been demonstrated that follicular phase may be shortened in older ovulatory women due to earlier dominant follicle selection. Moreover, low response may also be associated with accelerated luteinization of mature follicles $(28,29)$. The relatively high prevalence of poor responder patients may be a potential reason of advanced follicular maturation, and possibly premature ovulation. Therefore, particularly in patients with diminished ovarian reserve, flexible protocol may not be suitable, and early administration of dydrogesterone may be necessary to prevent premature ovulation. However, Turkgeldi et al. (18) recently reported that flexible PPOS protocol might be used as an alternative to the flexible GnRHant protocol in patients with diminished ovarian reserve. In this study, pituitary suppression by MPA was commenced as the estradiol level was $\geq 200 \mathrm{ng} / \mathrm{L}$ in contrast to the $300 \mathrm{ng} / \mathrm{L}$ threshold of estradiol in our study. A similar threshold level was used in the present study with our normal flexible GnRHant protocol since there is no clear cut-off for flexible PPOS protocols, and only one premature ovulation was encountered in the study group, which consisted of 27 patients. These findings suggest that dydrogesterone should be administered earlier but further research is required to confirm this suggestion.

In patients with premature ovulation, oocytes could be retrieved from 4 of 6 of them. It was also reported in previous studies that fertilization and live birth could be achieved from the oocytes of the smaller follicles as well as the oocytes collected from cul-de-sac after premature ovulation (30).

\section{Study limitation}

The main limitation of this study is its retrospective nature. However, a lack of difference between the demographic characteristics of both groups may decrease the risk of bias that may occur. Besides, while live birth rate is an important parameter in evaluating cycle success, pregnancy outcomes could not be assessed due to freeze-all strategy, and in particular, cryopreservation of the oocytes for fertility preservation in a not inconsiderable proportion of the patients.

\section{Conclusion}

Dydrogesterone can be used as an alternative to antagonist regimen in patients, where embryo transfer is not planned for the same cycle. However, particularly in patients with diminished ovarian reserve, early initiation of progesterone may be appropriate, owing to advanced and accelerated follicular maturation and due to the oral absorbtion pharmacokinetics of dydrogesterone. However, randomized controlled trials in particular populations are required to determine the most effective minimum dose and time of application to ensure treatment success.

Ethics Committee Approval: This study was approved by Institutional Review Board and Ethics Committee of Ankara University Faculty of Medicine (approval number: 20-1364-18).

Informed Consent: All patients gave informed consent prior to their treatment.

Peer-review: Externally peer-reviewed.

Author Contributions: Surgical and Medical Practices: G.D.D., G.B., E.T., H.G., M.S., Y.E.S., B.Ö., C.A., B.B., R.A., Mu.S.; Concept: G.D.D., G.B., E.T., H.G., Mu.S.; Design: G.D.D., G.B., E.T., H.G., M.S.; Data Collection or Processing: G.D.D., G.B., E.T., H.G.; Analysis or Interpretation: G.D.D., G.B., E.T., Mu.S.; Literature Search: G.D.D., E.T., H.G., M.S.; Writing: G.D.D., G.B., M.S.

Conflict of Interest: No conflict of interest is declared by the authors.

Financial Disclosure: The authors declared that this study received no financial support.

\section{References}

1. Kuang Y, Chen Q, Fu Y, Wang Y, Hong Q, Lyu Q, et al. Medroxyprogesterone acetate is an effective oral alternative for 
preventing premature luteinizing hormone surges in women undergoing controlled ovarian hyperstimulation for in vitro fertilization. Fertil Steril 2015; 104: 62-70.

2. Massin N. New stimulation regimens: endogenous and exogenous progesterone use to block the LH surge during ovarian stimulation for IVF. Hum Reprod Update 2017; 23: 211-20.

3. Wang Y, Kuang Y, Chen Q, Cai R. Gonadotropin-releasing hormone antagonist versus progestin for the prevention of premature luteinising hormone surges in poor responders undergoing in vitro fertilisation treatment: study protocol for a randomised controlled trial. Trials 2018; 19: 455.

4. Yu S, Long H, Chang HY, Liu Y, Gao H, Zhu J, et al. New application of dydrogesterone as a part of a progestin-primed ovarian stimulation protocol for IVF: a randomized controlled trial including 516 first IVF/ICSI cycles. Hum Reprod 2018; 33: 229-37.

5. Chen YM, Qi QR, Xie QZ, Yang YF, Xia Y, Zhou XD. Effect of progestinprimed ovarian stimulation protocol on outcomes of aged infertile women who failed to get pregnant in the first IVF/ ICSI Cycle: A self-controlled study. Curr Med Sci 2018; 38: 513-8.

6. Cobo A, Kuwayama M, Pérez S, Ruiz A, Pellicer A, Remohí J. Comparison of concomitant outcome achieved with fresh and cryopreserved donor oocytes vitrified by the Cryotop method. Fertil Steril 2008; 89: 1657-64.

7. Xiao ZN, Peng JL, Yang J, Xu WM. Flexible GnRH antagonist protocol versus progestin-primed ovarian stimulation (PPOS) protocol in patients with polycystic ovary syndrome: comparison of clinical outcomes and ovarian response. Curr Med Sci 2019; 39: 431-6.

8. Chen Q, Chai W, Wang Y, Cai R, Zhang S, Lu X, et al. Progestin vs. gonadotropin-releasing hormone antagonist for the prevention of premature luteinizing hormone surges in poor responders undergoing in vitro fertilization treatment: a randomized controlled trial. Front Endocrinol 2019; 10: 796.

9. Yildiz S, Turkgeldi E, Angun B, Eraslan A, Urman B, Ata B. Comparison of a novel flexible progestin primed ovarian stimulation protocol and the flexible gonadotropin-releasing hormone antagonist protocol for assisted reproductive technology. Fertil Steril 2019; 112: 677-83.

10. Grisendi V, Mastellari E, La Marca A. Ovarian reserve markers to identify poor responders in the context of poseidon classification. Front Endocrinol 2019; 10: 281.

11. Inaudi P, Barra V, Vellucci FL, Regini C, Luisi S. GnRH antagonist does not prevent premature luteinization and ovulation in stimulated cycles with gonadotropins for IVF: two case reports. Gynecol Endocrinol 2018; 34: 189-91.

12. Attardi B, Scott R, Pfaff D, Fink G. Facilitation or inhibition of the oestradiol-induced gonadotrophin surge in the immature female rat by progesterone: effects on pituitary responsiveness to gonadotrophin-releasing hormone (GnRH), GnRH self-priming and pituitary mRNAs for the progesterone receptor A and B isoforms. J Neuroendocrinol 2007; 19: 988-1000.

13. Evans MB, Parikh T, DeCherney AH, Csokmay JM, Healy MW, Hill MJ. Evaluation of the cost-effectiveness of ovulation suppression with progestins compared with GnRH analogs in assisted reproduction cycles. Reprod Biomed Online 2019; 38: 691-8.

14. Beguería R, García D, Vassena R, RodríguezA. Medroxyprogesterone acetate versus ganirelix in oocyte donation: a randomized controlled trial. Hum Reprod 2019; 34: 872-80.

15. Ata B, Capuzzo M, Turkgeldi E, Yildiz S, La Marca A. Progestins for pituitary suppression during ovarian stimulation for ART: a comprehensive and systematic review including meta-analyses. Hum Reprod Update 2021; 27: 48-66.
16. Wang Y, Chen Q, Wang N, Chen H, Lyu Q, Kuang Y. Controlled ovarian stimulation using medroxyprogesterone acetate and hMG in Patients with polycystic ovary syndrome treated for IVF: a doubleblind randomized crossover clinical trial. Medicine 2016; 95: e2939.

17. Huang P, Tang M, Qin A. Progestin-primed ovarian stimulation is a feasible method for poor ovarian responders undergoing in IVF/ ICSI compared to a GnRH antagonist protocol: A retrospective study. J Gynecol Obstet Hum Reprod 2019; 48: 99-102.

18. Turkgeldi E, Yildiz S, Cekic SG, Shakerian B, Keles I, Ata B. Effectiveness of the flexible progestin primed ovarian stimulation protocol compared to the flexible $\mathrm{GnRH}$ antagonist protocol in women with decreased ovarian reserve. Hum Fertil 2020: 1-7.

19. Wikström A, Green B, Johansson ED. The plasma concentration of medroxyprogesterone acetate and ovarian function during treatment with medroxyprogesterone acetate in 5 and $10 \mathrm{mg}$ doses. Acta Obstet Gynecol Scand 1984; 63: 163-8.

20. Dong J, Wang Y, Chai WR, Hong QQ, Wang NL, Sun LH, et al. The pregnancy outcome of progestin-primed ovarian stimulation using 4 versus $10 \mathrm{mg}$ of medroxyprogesterone acetate per day in infertile women undergoing in vitro fertilisation: a randomised controlled trial. BJOG 2017; 124: 1048-55.

21. Schindler AE, Campagnoli C, Druckmann R, Huber J, Pasqualini JR, Schweppe KW, et al. Classification and pharmacology of progestins. Maturitas 2008; 61: 171-80.

22. Rižner TL, Brožič P, Doucette C, Turek-Etienne T, Müller-Vieira U, Sonneveld E, et al. Selectivity and potency of the retroprogesterone dydrogesterone in vitro. Steroids 2011; 76: 607-15.

23. Bosch E, Valencia I, Escudero E, Crespo J, Simon C, Remohí J, et al. Premature luteinization during gonadotropin-releasing hormone antagonist cycles and its relationship with in vitro fertilization outcome. Fertil Steril 2003; 80: 1444-9.

24. Reichman DE, Zakarin L, Chao K, Meyer L, Davis OK, Rosenwaks Z. Diminished ovarian reserve is the predominant risk factor for gonadotropin-releasing hormone antagonist failure resulting in breakthrough luteinizing hormone surges in in vitro fertilization cycles. Fertil Steril 2014; 102: 99-102.

25. Lu X, Hong Q, Sun L, Chen Q, Fu Y, Ai A, et al. Dual trigger for final oocyte maturation improves the oocyte retrieval rate of suboptimal responders to gonadotropin-releasing hormone agonist. Fertil Steril 2016; 106: 1356-62.

26. Helmond F, Simons P, Hein P. The effects of progesterone on estrogen-induced luteinizing hormone and follicle-stimulating hormone release in the female rhesus monkey. Endocrinology 1980; 107: 478-85.

27. Helmond FA, Simons PA, Hein PR. Strength and duration characteristics of the facilitory and inhibitory effects of progesterone on the estrogen-induced gonadotropin surge in the female rhesus monkey. Endocrinology 1981; 108: 1837-42.

28. Klein NA, Harper AJ, Houmard BS, Sluss PM, Soules MR. Is the short follicular phase in older women secondary to advanced or accelerated dominant follicle development? J Clin Endocrinol Metab 2002; 87: 5746-50.

29. Luborsky J, Thiruppathi P, Rivnay B, Roussev R, Coulam C, Radwanska E. Evidence for different aetiologies of low estradiol response to FSH: age-related accelerated luteinization of follicles or presence of ovarian autoantibodies. Hum Reprod 2002; 17: 26419.

30. Wu FS, Lee RK, Hwu YM. Encountering premature ovulation during controlled ovarian hyperstimulation in IVF/ICSI cycles. Taiwan J Obstet Gynecol 2012; 51: 256-9. 